\title{
Correlation and Capacity of Measured Multi-user MIMO Channels
}

\author{
Florian Kaltenberger, David Gesbert, Raymond Knopp \\ Institute Eurecom \\ 2229, Route des Cretes - B.P. 193 \\ 06904 Sophia Antipolis, France
}

\author{
Marios Kountouris \\ Wireless Networking and Communications Group \\ The University of Texas at Austin \\ Austin, TX 78712, USA
}

\begin{abstract}
In multi-user multiple-input multiple-output (MUMIMO) systems, spatial multiplexing can be employed to increase the throughput without the need for multiple antennas and expensive signal processing at the user equipments. In theory, MU-MIMO is also more immune to most of propagation limitations plaguing single-user MIMO (SU-MIMO) systems, such as channel rank loss or antenna correlation. However, in this paper we show that this is not always true. We compare the capacity and the correlation of measured MU-MIMO channels for both outdoor and indoor scenarios. The measurement data has been acquired using Eurecom's MIMO Openair Sounder (EMOS). The EMOS can perform real-time MIMO channel measurements synchronously over multiple users. The results show that in most scenarios MU-MIMO provides a higher throughput than SU-MIMO also in the measured channels. However, in outdoor scenarios with a line of sight, the capacity drops significantly when the users are close together, due to high correlation at the transmitter side of the channel. In such a case, the performance of SU-MIMO and MU-MIMO is comparable.
\end{abstract}

\section{INTRODUCTION}

We study the downlink (or broadcast) channel of a wideband multi-user multiple-input multiple-output (MU-MIMO) system in which there are multiple antennas at the base-station (BS) and possibly multiple antennas at the user equipment (UE).

Information theory reveals that if the channel is fully known at the transmitter and the receiver, the optimum transmit strategy for the MU-MIMO broadcast channel involves a theoretical pre-interference cancellation technique known as dirty paper coding (DPC) combined with an implicit user scheduling and power loading algorithm [1], [2]. Compared to a single-user MIMO (SU-MIMO) time division multiple access (TDMA) system, DPC can bring a theoretical performance gain of up to $\max (\min (M / N, K), 1)$ in an independent and identically distributed (i.i.d.) Rayleigh fading channel, where $M$ and $N$ is the number of transmit antennas and receive antennas respectively and $K$ is the number of users [3]. However, DPC is very computationally expensive and thus simpler, sub-optimal transmit strategies have been proposed.

The assumption of an i.i.d. channel is often justified using the argument that the users are spatially separated and thus the signals arriving at different users will be independent even in the presence of a line of sight (LOS) component [2]. However, it was shown in [4] that the throughput in the measured

This research was supported by the project PACAM with SFR, the EC under FP7 Network of Excellence project NEWCOM++ and Eurecom. channels is worse than the one in i.i.d. channels. In this paper we investigate the spatial correlation of measured MUMIMO channels for both outdoor and indoor scenarios. We also compare the performance of different linear MU-MIMO precoding schemes, such as zero-forcing $(\mathrm{ZF})$ and regularized inversion (also called MMSE precoder) [5].

Realistic MU-MIMO channel measurements have been obtained using Eurecom's MIMO Openair Sounder (EMOS) [6]. The EMOS can perform real-time channel measurements synchronously over multiple users moving at vehicular speed. For this paper, we have used four transmit antennas and four users with one antennas each. The measured channels are used to calculate the capacity offline, assuming a perfect feedback channel. To the best of our knowledge, no such comparison based on real MU channel measurements has been reported. Real indoor channel measurements have been used in [7] for the evaluation of the proposed MU-MIMO scheme. Real outdoor channel measurements have been used in [8] to study limited feedback. However, the channel measurements were obtained with one receiver at different times and not synchronously as in our measurements.

The paper is organized as follows. We introduce the signal model in Section II. The performance metrics for the evaluation of the channel measurements are presented in III. In Section IV we describe the EMOS in some more detail and explain how the channel measurements are performed. In Section V the measurement campaign is described and results are discussed. We finally give conclusions in Section VI.

\section{SySTEM MODEL}

We consider a multi-user, multi-antenna downlink channel in which a BS equipped with $M$ antennas communicates with $K \leq M$ terminals, each equipped with $N$ antennas. The received signal $\mathbf{y}_{k, m, q} \in \mathbb{C}^{N \times 1}$ of the $k$-th user at time $m$ and frequency $q$ is mathematically described as

$$
\mathbf{y}_{k, m, q}=\mathbf{H}_{k, m, q} \mathbf{x}_{m, q}+\mathbf{n}_{k, m, q} \quad \text { for } k=1, \ldots, K
$$

where $\mathbf{H}_{k, m, q} \in \mathbb{C}^{N \times M}$ represents the $k$-th user channel response at time $m$ and frequency $q, \mathbf{x}_{m, q} \in \mathbb{C}^{M \times 1}$ is the vector of transmitted symbols at time $m$ and frequency $q$, and $\mathbf{n}_{k, m, q} \in \mathbb{C}^{N \times 1}$ is i.i.d. circularly symmetric additive complex Gaussian noise with zero mean and variance $\sigma^{2}, \forall k$. We assume that each of the receivers has perfect and instantaneous 
knowledge of its own channel. The transmitter is subject to an average power constraint, i.e., $\mathbb{E}\left\{\mathbf{x}_{m, q}^{H} \mathbf{x}_{m, q}\right\} \leq P$, which implies that the total transmit power is not dependent on the number of transmit antennas. For notation convenience, in the following sections we drop the time and frequency indices.

\section{CAPACITY AND CORRELATION}

To analyze the measured MU-MIMO channels we use the following performance metrics: sum rate capacity under DPC, sum rate capacity under MU-MIMO linear precoding, sum rate capacity under SU-MIMO TDMA, correlation at the receiver, correlation at the transmitter.

\section{A. Dirty Paper Coding}

From the results in [1], [9], [10], the sum capacity of the MU-MIMO downlink channel can be expressed by the following maximization:

$$
\begin{aligned}
& \mathcal{C}_{\mathrm{BC}}\left(\mathbf{H}_{1}, \ldots, \mathbf{H}_{K}, P\right)= \\
& \max _{\boldsymbol{\Sigma}_{k} \geq 0, \sum_{k=1}^{K} \operatorname{tr}\left(\boldsymbol{\Sigma}_{k}\right) \leq P} \sum_{k=1}^{K} \log _{2} \frac{\left|\mathbf{I}+\mathbf{H}_{k}\left(\sum_{j=1}^{K} \boldsymbol{\Sigma}_{j}\right) \mathbf{H}_{k}^{H}\right|}{\left|\mathbf{I}+\mathbf{H}_{k}\left(\sum_{j \neq k} \boldsymbol{\Sigma}_{j}\right) \mathbf{H}_{k}^{H}\right|},
\end{aligned}
$$

where the maximization is over the set of all positive semidefinite transmit covariance matrices $\Sigma_{k}, k=1, \ldots, K$. The objective function of the maximization in (2) is a concave function of the covariance matrices, making it very difficult to deal with. Fortunately, due to the MAC-BC duality, the sum rate capacity of the MIMO BC is equal to the sum rate capacity of the dual MAC with power constraint $P$

$$
\begin{gathered}
\mathcal{C}_{\mathrm{BC}}\left(\mathbf{H}_{1}, \ldots, \mathbf{H}_{K}, P\right)=\mathcal{C}_{\mathrm{MAC}}\left(\mathbf{H}_{1}, \ldots, \mathbf{H}_{K}, P\right)= \\
\max _{\mathbf{Q}_{k} \geq 0, \sum_{k=1}^{K} \operatorname{tr}\left(\mathbf{Q}_{k}\right) \leq P} \log _{2}\left|\mathbf{I}+\sum_{k=1}^{K} \mathbf{H}_{k} \mathbf{Q}_{k} \mathbf{H}_{k}^{H}\right|
\end{gathered}
$$

where each of the matrices $\mathbf{Q}_{i}$ is a positive semidefinite covariance matrix. Since (3) involves the maximization of a concave function, efficient numerical algorithms exist. In this paper, we use the specialized algorithm developed in [11] to calculate $\mathcal{C}_{\mathrm{BC}}\left(\mathbf{H}_{1}, \ldots, \mathbf{H}_{K}, P\right)$.

It has been shown [12] that the sum rate capacity given in Equation (3) is actually achieved by using DPC. However, DPC is very complex and difficult to implement. Thus we also study linear precoding schemes in the next section.

\section{B. Linear Precoding Sum Rate}

Let $\mathbf{s}_{k} \in \mathbb{C}^{N \times 1}$ denote the $k$-th user transmit symbol vector. Under linear precoding, the transmitter multiplies the data symbol for each user $k$ by a precoding matrix $\mathbf{W}_{k} \in$ $\mathbb{C}^{M \times N}$ so that the transmitted signal is a linear function $\mathbf{x}=\sum_{k=1}^{K} \mathbf{W}_{k} \mathbf{s}_{k}$. The resulting received signal vector for user $k$ is given by

$$
\mathbf{y}_{k}=\mathbf{H}_{k} \mathbf{W}_{k} \mathbf{s}_{k}+\sum_{j \neq k} \mathbf{H}_{k} \mathbf{W}_{j} \mathbf{s}_{j}+\mathbf{n}_{k}
$$

where the second-term in (4) represents the multi-user interference. We assume that each user will decode $S \leq N$ streams that constitute its data. The goal of linear precoding is to design $\left\{\mathbf{W}_{k}\right\}_{k=1}^{K}$ based on the channel matrix knowledge, so a given performance metric is maximized for each stream.

1) Zero-Forcing Precoding (Channel Inversion): For ease of exposition, we assume $N=1$ and we define $\mathbf{H}=$ $\left[\mathbf{h}_{1}^{T} \ldots \mathbf{h}_{K}^{T}\right]^{T}$. The unit-norm beamforming vector of user $k$ is denoted as $\mathbf{w}_{k} \in \mathbb{C}^{M \times 1}, k=1, \ldots, K$.

A standard suboptimal approach providing a promising tradeoff between complexity and performance is zero-forcing precoding, also known as channel inversion. In $\mathrm{ZF}$, the precoder is designed to achieve zero interference between the users, i.e., $\mathbf{h}_{k} \mathbf{w}_{j}=0$ for $j \neq k$. The $\mathrm{ZF}$ precoding matrix is given by the Moore-Penrose pseudoinverse of $\mathbf{H}$

$$
\mathbf{W}=\mathbf{H}^{\dagger}=\mathbf{H}^{H}\left(\mathbf{H} \mathbf{H}^{H}\right)^{-1},
$$

where $\mathbf{w}_{k}$ is obtained by normalizing the $k$-th column of $\mathbf{W}$.

Assuming equal power allocation over the users, the achievable sum rate is given by

$$
\mathcal{R}_{\mathrm{ZF}}=\sum_{k=1}^{K} \log _{2}\left(1+\frac{P}{K \sigma^{2}}\left|\mathbf{h}_{k} \mathbf{w}_{k}\right|^{2}\right) .
$$

When the channel is ill-conditioned, at least one of the singular values of $\left(\mathbf{H H}^{H}\right)^{-1}$ is very large, resulting in a very low SNR at the receivers. Note also that $\mathrm{ZF}$ precoding - in contrast to $\mathrm{ZF}$ (least-squares) equalization at the receive side which causes noise enhancement when the channel is nearly rank deficientincurs an excess transmission power penalty. Therefore, the capacity of channel inversion with no user selection does not increase linearly with $M$, unlike the optimum capacity.

2) MMSE Precoding (Regularized Channel Inversion): For rank-deficient channels, the performance of ZF precoding can be improved by a regularization of the pseudo-inverse, which can be expressed as:

$$
\mathbf{W}=\mathbf{H}^{H}\left(\mathbf{H} \mathbf{H}^{H}+\beta \mathbf{I}\right)^{-1},
$$

where $\beta$ is a regularization factor. The above scheme is often referred to as Minimum Mean Square-Error (MMSE) precoding due to the analogous with MMSE beamforming weight design criterion if the noise is spatially white. The achievable throughput is given by

$$
\mathcal{R}_{\mathrm{MMSE}}=\sum_{k=1}^{K} \log _{2}\left(1+\frac{\left|\mathbf{h}_{k} \mathbf{w}_{k}\right|^{2}}{\sum_{j \neq k}\left|\mathbf{h}_{k} \mathbf{w}_{j}\right|^{2}+K \sigma^{2} / P}\right),
$$

where $\mathbf{w}_{k}$ is the normalized $k$-th column of the precoder given in (7).

Similarly to MMSE equalization, a non-zero $\beta$ value results in a measured amount of multi-user interference. The amount of interference is determined by $\beta>0$ and an optimal tradeoff between the condition of the channel matrix inverse and the amount of crosstalk ought to be found. In practice, the regularization factor is commonly chosen as $\beta=M \sigma^{2} / P$ motivated by the results in [5] that show that it approximately maximizes the SINR at each receiver, and leads to linear 


\begin{tabular}{|c|c|}
\hline Parameter & Value \\
\hline Center Frequency & $1917.6 \mathrm{MHz}$ \\
Bandwidth & $4.8 \mathrm{MHz}$ \\
BS Transmit Power & $30 \mathrm{dBm}$ \\
Number of Antennas at BS & $4(2$ cross polarized $)$ \\
Number of UE & 4 \\
Number of Antennas at UE & 2 \\
Number of Subcarriers & 160 \\
\hline
\end{tabular}

TABLE I

EMOS PARAMETERS

capacity growth with $M$. The performance of MMSE is certainly significantly better at low SNR and converges to that of ZF precoding at high SNR. However, MMSE does not provide parallel and orthogonal channels and thus power allocation techniques cannot be performed in a straightforward manner.

\section{Time Division Multiple Access Sum Rate}

The capacity of a single user $k$ is given by

$$
\mathcal{C}_{\text {SU-MIMO }}\left(\mathbf{H}_{k}, P\right)=\max _{\mathbf{Q}_{k} \geq 0, \operatorname{tr}\left(\mathbf{Q}_{k}\right) \leq P} \log _{2}\left|\mathbf{I}+\mathbf{H}_{k} \mathbf{Q}_{k} \mathbf{H}_{k}^{H}\right| .
$$

The maximum is achieved by choosing the covariance matrix $\mathbf{Q}_{k}$ to be along the eigenvectors of the matrix $\mathbf{H}_{k} \mathbf{H}_{k}^{H}$ and by choosing the eigenvalues according to the water filling procedure [13]. The maximum sum rate capacity is achieved by transmitting to the user with the largest single-user capacity. However, in this paper we assume that all users are served fairly proportional in a round robin fashion, i. e., we treat each $\mathbf{H}_{k}$ as a different realization.

\section{Transmit and Receive Correlation}

The transmit and receive correlation matrices of the MUMIMO channel $\mathbf{H}$ are defined as

$$
\begin{aligned}
& \mathbf{R}_{\mathrm{Tx}}=\mathbb{E}\left\{\mathbf{H}^{H} \mathbf{H}\right\}, \\
& \mathbf{R}_{\mathrm{Rx}}=\mathbb{E}\left\{\mathbf{H H}^{H}\right\} .
\end{aligned}
$$

For the measurements we calculate the expectation by taking the mean of the channel over all frequencies $q$ and all frames $m$ in one measurement.

$\mathbf{R}_{\mathrm{Tx}}$ and $\mathbf{R}_{\mathrm{Rx}}$ give insight to what extent the signals leaving the different transmit antennas and the signals arriving at the different receive antennas respectively are correlated. Note that in general $\mathbf{R}_{\mathrm{Tx}}$ and $\mathbf{R}_{\mathrm{Rx}}$ do not fully characterize the second order statistics of the channel [14]. However, they are more intuitively and sufficient for the purpose of this paper.

\section{The EMOS Multi-USER Platform}

\section{A. Hardware Description}

The Eurecom MIMO Openair Sounder (EMOS) is based on the OpenAir hardware/software development platform at Eurecom. The platform consists of a BS that continuously sends a signaling frame, and one or more UEs that receive the frames to estimate the channel. For the BS, an ordinary server PC with four PLATON data acquisition cards (see Fig. $1(\mathrm{a})$ ) is employed along with a Powerwave $3 \mathrm{G}$ broadband

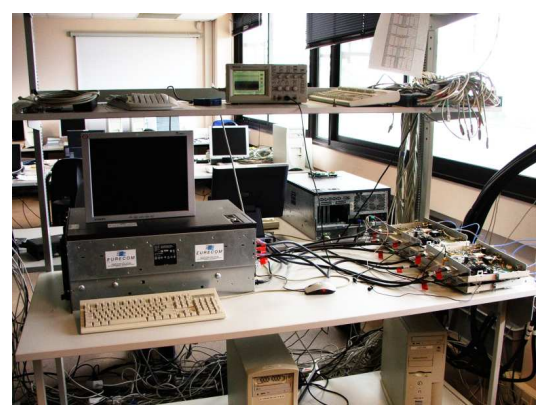

(a) Server PC with PLATON boards

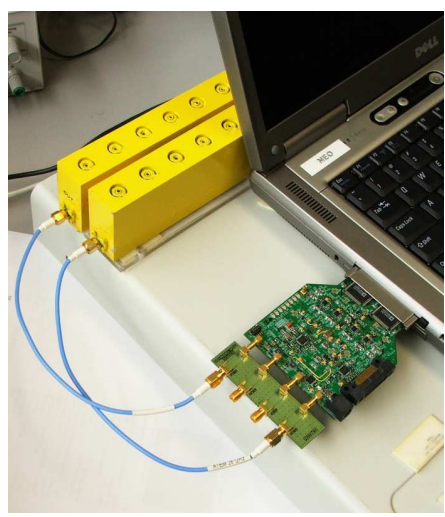

(c) Dual-RF CardBus/PCMCIA Card

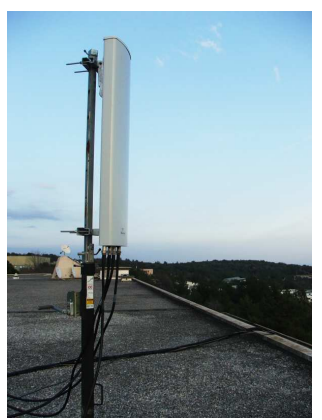

(b) Powerwave Antenna

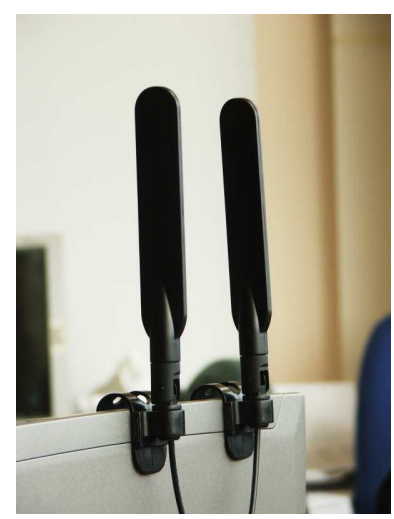

(d) Panorama Antennas
Fig. 1. EMOS base-station and user equipment [6]

antenna (part no. 7760.00) composed of four elements which are arranged in two cross-polarized pairs (see Fig. 1(b)). The UEs consist of an ordinary laptop computer with Eurecom's dual-RF CardBus/PCMCIA data acquisition card (see Fig. 1(c)) and two clip-on 3G Panorama Antennas (part no. TCLIPDE3G, see Fig. 1(d)). The platform is designed for a full software-radio implementation, in the sense that all protocol layers run on the host PCs under the control of a Linux real time operation system.

\section{B. Sounding Signal}

The EMOS is using an OFDM modulated sounding sequence. One transmit frame is $2.667 \mathrm{~ms}$ long and consists of a synchronization symbol ( $\mathrm{SCH})$, a broadcast data channel (BCH) comprising 7 OFDM symbols, a guard interval, and 48 pilot symbols used for channel estimation (see Fig. 2). The pilot symbols are taken from a pseudo-random QPSK sequence defined in the frequency domain. The subcarriers of the pilot symbols are multiplexed over the four transmit antennas to ensure orthogonality in the spatial domain. The $\mathrm{BCH}$ contains the frame number of the transmitted frame that is used for synchronization among the UEs.

\section{Channel Estimation Procedure}

Each UE first synchronizes to the BS using the SCH. It then tries to decode the data in the $\mathrm{BCH}$. If the $\mathrm{BCH}$ can be decoded successfully, then the channel estimation procedure is started. The channel estimation procedure consists of two 


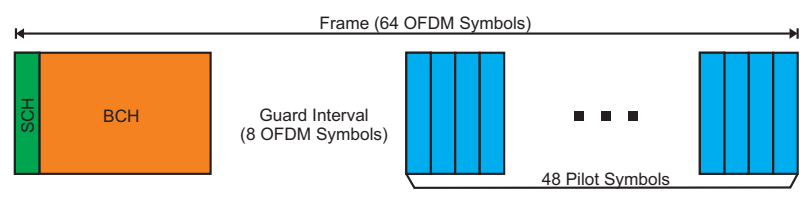

Fig. 2. Frame structure of the OFDM Sounding Sequence.

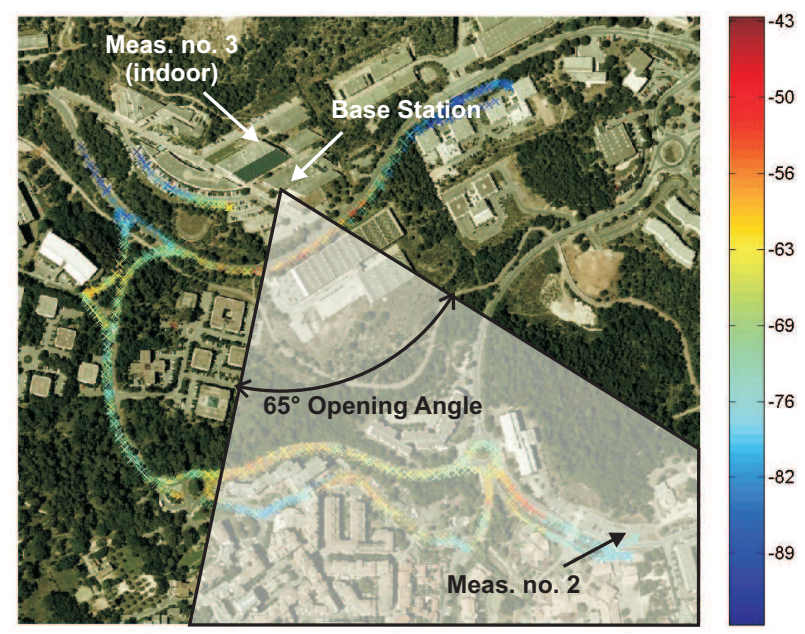

Fig. 3. Map of the measurement scenario. The position and the opening angle of the BS antenna are also indicated. In the first measurement the users were driving in cars along the indicated routes (the colors show the received signal strength in $\mathrm{dBm}$ along the routes). In the second and third measurement the users were close together at the indicated positions.

steps. Firstly, the pilot symbols are derotated with respect to the first pilot symbol to reduce the phase-shift noise generated by the dual-RF CardBus/PCMCIA card. Secondly, the pilot symbols are averaged to increase the measurement SNR. The estimated MIMO channel is finally stored to disk. For a more detailed description of the channel estimation see [6].

\section{Multi-user Measurement Procedure}

In order to conduct multi-user measurements, all the UEs need to be frame-synchronized to the BS. This is achieved by storing the frame number encoded in the $\mathrm{BCH}$ along with the measured channel at the UEs. This way, the measured channels can be aligned for later evaluations. The frame number is also used to synchronize the data acquisition between UEs. One measurement run (file) starts every 22.500 frames $(60 \mathrm{sec})$ and is exactly 18.750 frames $(50 \mathrm{sec})$ long.

\section{Measurements And Results}

\section{A. Measurement Description}

For the presentation in this paper we selected three different representative measurement runs. Two of the measurements were conducted outdoors in the vicinity of the Eurecom institute. The scenario is characterized by a semi-urban hilly terrain, composed by short buildings and vegetation with a predominantly present LOS. Fig. 3 shows a map of the environment. The BS is located at the roof of Eurecom's southmost building. The antenna is directed towards Garbejaire, a small nearby village. In the first measurement, the UEs were placed

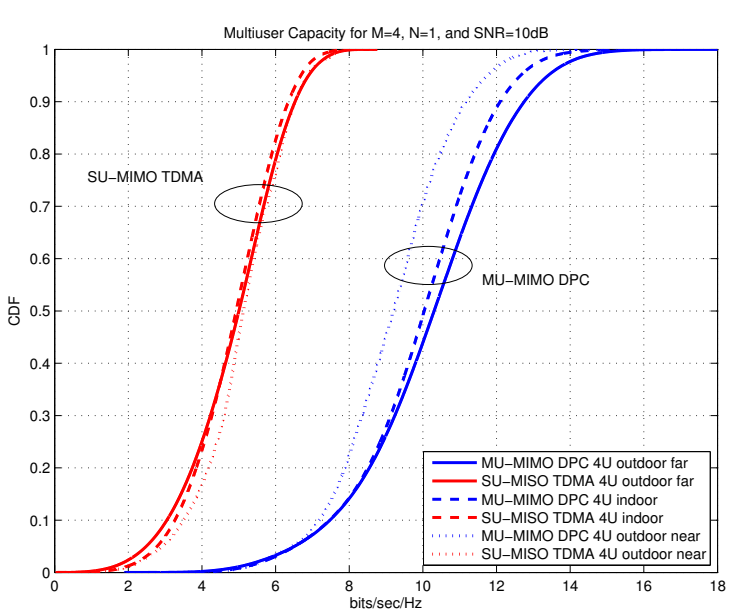

Fig. 4. CDF of the sum rate of SU-MIMO TDMA compared to MU-MIMO with DPC for all three measurements (meas. no. 1 = "outdoor far", meas. no. $2=$ "outdoor near", meas. no. 3 = "indoor"). The average SNR is fixed to $10 \mathrm{~dB}$ for each user.

inside standard passenger cars which were beeing driven along the routes shown in Fig. 3, keeping a large distance. In the second measurement, the cars were parked close together on a parking space indicated in the figure. The third measurement was conducted indoors in the neighboring building. The indoor scenario is characterized by strong reflections (the buildings is actually located behind the main lobe of the antenna) and thus there is no LOS. The users were all in the same room, moving around slowly.

\section{B. Results}

For all evaluations in this paper, we use only the first antenna at the UEs. Further, to ensure a constant average SNR of $10 \mathrm{~dB}$ at the UEs, the channel of every user is normalized over the whole measurement run (about $50 \mathrm{sec}$ ). Firstly, we compare the performance of MU-MIMO using DPC, ZF precoding, and MMSE precoding as well as SUMIMO TDMA based on the empirical cumulative density function (CDF) of the sum rate (Equations (3), (6), (8), and (9)). The results are plotted in Figures 4 and 5. Secondly we compare the transmit and receive correlation matrices $\mathbf{R}_{\mathrm{Tx}}$ and $\mathbf{R}_{\mathrm{Rx}}$ (cf. Equations (10) and (11)). In Fig. 6 the matrices are represented graphically using different shades of gray to indicate the absolute value of the matrix entry (white $=$ no correlation, black = high correlation).

\section{Discussion}

It can be seen from Fig. 4 that MU-MIMO DPC as well as SU-MISO TDMA do not show a very high variability with respect to the three different measurements. However, the linear MU-MIMO precoding schemes (see Fig. 5) are very sensitive to the channel conditions. Especially the performance of the ZF precoder drops significantly in the outdoor scenario where the users are close together. In the indoor scenario and the other outdoor scenario where all users are well 


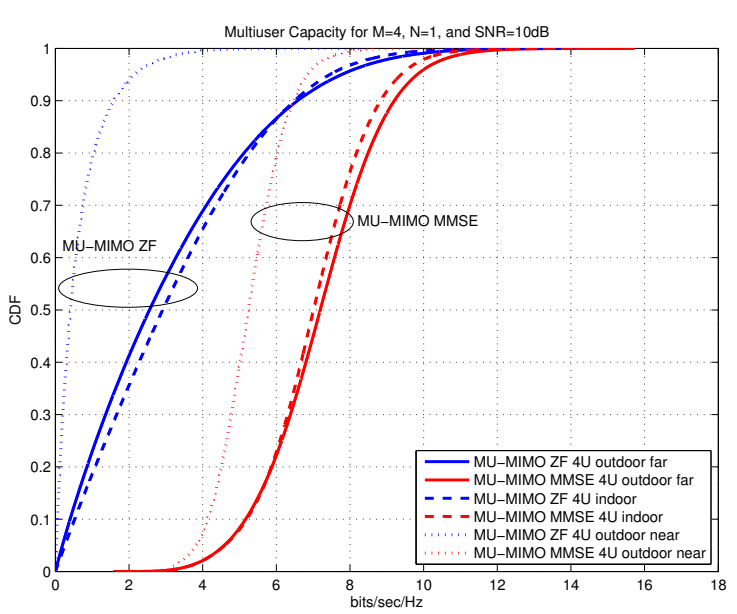

Fig. 5. CDF of the sum rate of MU-MIMO with ZF and MMSE precoding for all three measurements (meas. no. $1=$ "outdoor far", meas. no. $2=$ "outdoor near", meas. no. 3 = "indoor"). The average SNR is fixed to $10 \mathrm{~dB}$ for each user.

separated, the performance of the linear MU-MIMO schemes is comparable.

These facts can be explained by looking at the channel correlation in the different scenarios (Fig. 6). It can be seen that in all the scenarios, almost no correlation can be measured at the receive side between the different users. However, at the transmit side, the situation is different in all three scenarios. In the outdoor case with spatially separated users, there is just a little correlation between cross polarized components of the transmit antenna. In the indoor scenario, there is slightly, but not significantly more correlation. In the outdoor scenario where the users are located close together however, the correlation rather strong.

When the channel is strongly correlated it means that the channel matrix is ill-conditioned. Thus at least one of the singular values of $\left(\mathbf{H H}^{H}\right)^{-1}$ is very large, resulting in a very low SNR at the receivers, when ZF precoding is used. The MMSE precoder can alleviate this problem, but still suffers from the high correlation at the transmitter.

\section{CONCLUSIONS}

We have presented capacity and correlation analysis of measured MU-MIMO channels. The data was acquired using Eurecom's MU-MIMO channel sounder EMOS. We have shown that linear precoding schemes are very sensitive to the spatial correlation of the channel. In particular, the performance of a $\mathrm{ZF}$ precoder drops significantly in outdoor scenarios, when the users are close together. It was found out that this drop in performance is due to the strong channel correlation at the transmit side in those scenarios. This finding is contradicts the common assumption of the MU-MIMO channel being i.i.d. However, it is true, that in all the measured MU-MIMO channels, there is no correlation at the receive side. We conclude that to ensure good performance of MUMIMO it is essential to do user selection at the base station. The users should be selected in such a way that their channels
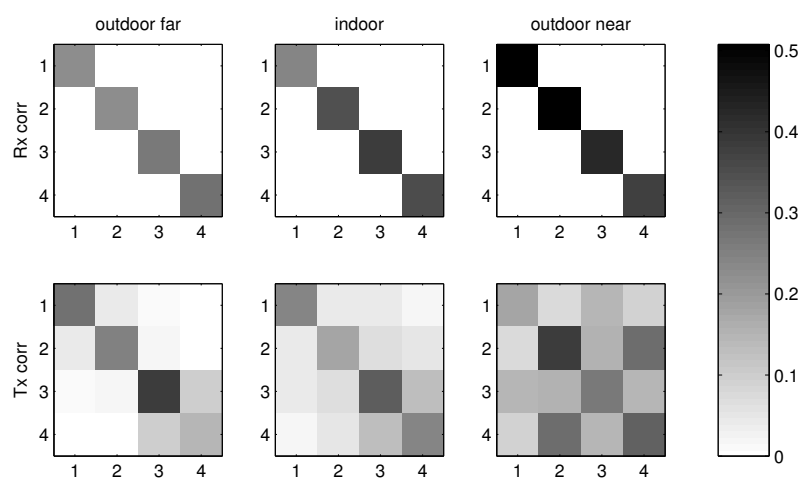

Fig. 6. Absolute values of the Tx and Rx correlation matrices.

are as orthogonal as possible. Otherwise it is not worth to do MU-MIMO linear precoding, since its performance is then comparable to SU-MIMO TDMA.

\section{REFERENCES}

[1] G. Caire and S. Shamai (Shitz), "On the achievable throughput of a multiantenna Gaussian broadcast channel," IEEE Trans. Inf. Theory, vol. 49, no. 7, pp. 1691-1706, Jul. 2003.

[2] D. Gesbert, M. Kountouris, R. W. Heath, Jr., C. B. Chae, and T. Sälzer, "From single user to multiuser communications: Shifting the MIMO paradigm," IEEE Signal Process. Mag., vol. 24, no. 5, pp. 36-46, Sep. 2007

[3] N. Jindal and A. Goldsmith, "Dirty-paper coding versus TDMA for MIMO broadcast channels," IEEE Trans. Inf. Theory, vol. 51, no. 5, pp. 1783-1794, May 2005.

[4] F. Kaltenberger, L. S. Cardoso, M. Kountouris, R. Knopp, and D. Gesbert, "Capacity of linear multi-user MIMO precoding schemes with measured channel data," in Proc. IEEE Intl. Workshop on Signal Processing Advances in Wireless Communications (SPAWC), Recife, Brazil, Jul. 2008.

[5] C. B. Peel, B. M. Hochwald, and A. L. Swindlehurst, "A vector-perturbation technique for near-capacity multiantenna multiuser communication-part I: channel inversion and regularization," IEEE Trans. Commun., vol. 53, no. 1, pp. 195-202, Jan. 2005.

[6] R. de Lacerda, L. S. Cardoso, R. Knopp, M. Debbah, and D. Gesbert, "EMOS platform: real-time capacity estimation of MIMO channels in the UMTS-TDD band," in Proc. International Symposium on Wireless Communication Systems (IWCS), Trondheim, Norway, Oct. 2007.

[7] G. Bauch, J. Anderson, C. Guthy, M. Herdin, J. Nielsen, J. A. Nossek, P. Tejera, and W. Utschick, "Multiuser MIMO channel measurements and performance in a large office environment," in Proc. IEEE Wireless Comm. and Net. Conf., Hong Kong, Mar. 2007, pp. 1900-1905.

[8] G. W. K. Colman and T. J. Willink, "Limited feedback precoding in realistic MIMO channel conditions limited feedback precoding in realistic MIMO channel conditions," in Proc. IEEE Int. Conf. on Comm. (ICC), Glasgow, Scotland, Jun. 2007, pp. 4363-4368.

[9] W. Yu and J. M. Cioffi, "Sum capacity of Gaussian vector broadcast channels," IEEE Trans. Inf. Theory, vol. 50, no. 9, pp. 1875-1892, Sep. 2004.

[10] P. Viswanath and D. Tse, "Sum capacity of the vector Gaussian broadcast channel and uplink-downlink duality," IEEE Trans. Inf. Theory, vol. 49, no. 8, pp. 1912-1921, Aug. 2003.

[11] N. Jindal, W. Rhee, S. Vishwanath, S. Jafar, and A. Goldsmith, "Sum power iterative water-filling for multi-antenna Gaussian broadcast channels," IEEE Trans. Inf. Theory, vol. 51, no. 4, pp. 1570-1580, Apr. 2005.

[12] H. Weingarten, Y. Steinberg, and S. Shamai (Shitz), "The capacity region of the gaussian multiple-input multiple-output broadcast channel," IEEE Trans. Inf. Theory, vol. 52, no. 9, pp. 3936-3964, Sep. 2006.

[13] E. Telatar, "Capacity of multi-antenna Gaussian channels," European Transactions on Telecommunications, vol. 10, pp. 585-595, 1999.

[14] H. Ozcelik, M. Herdin, W. Weichselberger, J. Wallace, and E. Bonek, "Deficiencies of "Kronecker" MIMO radio channel model," IEE Electronic Letters, vol. 39, no. 16, pp. 1209-1210, August 2003. 